\title{
Child obesity cut-offs as derived from parental perceptions:
}

\author{
cross-sectional questionnaire
}

\begin{abstract}
\section{Background}

Overweight children are at an increased risk of premature mortality and disease in adulthood. Parental perceptions and clinical definitions of child obesity differ, which may lessen the effectiveness of interventions to address obesity in the home setting. The extent to which parental and objective weight status cut-offs diverge has not been documented.

\section{Aim}

To compare parental perceived and objectively derived assessment of underweight, healthy weight, and overweight in English children, and to identify sociodemographic characteristics that predict parental under- or overestimation of a child's weight status.
\end{abstract}

\section{Design and setting}

Cross-sectional questionnaire completed by parents linked with objective measurement of height and weight by school nurses, in English children from five regions aged 4-5 and 10-11 years old.

\section{Method}

Parental derived cut-offs for under- and overweight were derived from a multinomial model of parental classification of their own child's weight status against school nurse measured body mass index (BMI) centile.

\section{Results}

Measured BMI centile was matched with parent classification of weight status in 2976 children. Parents become more likely to classify their children as underweight when they are at the 0.8 th centile or below, and overweight at the 99.7 th centile or above. Parents were more likely to underestimate a child's weight if the child was black or South Asian, male, more deprived, or the child was older. These values differ greatly from the BMI centile cut-offs for underweight (2nd centile) and overweight (85th).

\section{Conclusion}

Clinical and parental classifications of obesity are divergent at extremes of the weight spectrum.

\section{Keywords}

body mass index; child; cross-sectional studies; female; humans; male; obesity; parents; preschool; primary care.

\section{INTRODUCTION}

The prevalence of childhood obesity has increased significantly in recent years. ${ }^{1-3}$ In the 2012-2013 school year, 33\% of Year 6 pupils (age 10-11 years) in England were overweight or obese. ${ }^{4}$ Overweight children and adolescents are at an increased risk of cardiometabolic disease and premature mortality in adulthood. ${ }^{5}$ However, evidence from systematic literature reviews has consistently found that only $40-50 \%$ of parents can identify when their child is overweight. ${ }^{6.7}$ The lack of accurate parental perception of a child's weight status may limit the effectiveness of interventions aimed at primary prevention of child obesity.

The National Child Measurement Programme (NCMP) measures the heights and weights of all children in reception lage 4-5 years) and Year 6 laged 10-11 years) at state schools in England. ${ }^{4}$ Local authorities are encouraged to provide written weight status feedback to the children's parents, as well as telephone or in-person feedback to children that are overweight. ${ }^{4}$ Standard population body mass index (BMI) cutoffs used for NCMP surveillance are the 2nd centile for underweight, 85th centile for overweight, and 95th centile for very overweight; the NCMP uses more stringent clinical cut-offs of 2 nd centile for

JA Black, MPhil, PhD student; MH Park, PhD, research fellow; J Gregson, PhD, research fellow; S Kinra, MBBS, PhD, reader in clinical epidemiology, Department of Non-communicable Disease Epidemiology; AS Kessel, MBBS, MPhil, PhD, professor, Faculty of Public Health and Policy, London School of Hygiene \& Tropical Medicine, London. CL Falconer, PhD, research associate, School of Oral and Dental Sciences, University of Bristol, Bristol. B White, MBBS, consultant in adolescent diabetes; RM Viner, MBBS, PhD, professor, Department of General and Adolescent Paediatrics, Institute of Child Health, University College London, London. S Saxena, MD, reader in primary care, Department of Primary Care and Public Health, underweight, 91st centile for overweight, and 98th centile for very overweight in its feedback to parents. ${ }^{8}$

Child obesity cut-offs represent points where the deviation in BMI from a reference population of British children, collected between 1978 and 1990, is assumed extreme enough to infer membership of a different weight status group. ${ }^{9}$ Parents are known to misclassify their child's weight status,, 610 and little is known about what weight status cut-offs would look like if they were derived from parental perceptions of their child's weight status rather than expert opinion based on objective assessment of weight status. As parents may be less likely to act if they do not perceive their child's overweight as a problem, quantification of the discrepancy between perceived weight status by the parent and objectively measured weight status may help to explore the potential impact of parental weight misclassification on effectiveness of public health interventions aimed at reducing the prevalence of child obesity.

\section{Aims}

This study aimed to:

- compare parental-perceived and objectively-derived assessment of

Imperial College London, London.

\section{Address for correspondence}

James A Black, Department of

Non-communicable Disease Epidemiology, London School of Hygiene \& Tropical Medicine Keppel Street, London WC1E 7HT, UK.

E-mail: james.blackacantab.net

Submitted: 2 April 2014; Editor's response:

18 July 2014; final acceptance: 9 September 2014 (CBritish Journal of General Practice

This is the full-length article (published online 30 Mar 2015) of an abridged version published in print. Cite this article as: Br J Gen Pract 2015; DOI: 10.3399/bjgp15X684385 


\section{How this fits in}

Parental perceptions and clinical definitions of child obesity are known to diverge; however, the extent of the discrepancy has not been documented. This study characterises parental classifications of obesity and identifies sociodemographic characteristics that predict misclassification. Also, BMI centile cut-offs for weight status are established as derived from parental perceptions.

underweight, healthy weight, or overweight; and

- identify socidemographic characteristics that predict parental under- or overestimation of a child's weight status.

\section{METHOD}

The NCMP is an England-wide programme, which aims at measuring the heights and weights of every child in reception and Year 6 at state schools. Parents of all children enrolled in the NCMP in the 2010 2011 school year across the following five primary care trusts (PCTs) were invited to participate: Redbridge, Islington, West Essex, Bath and North East Somerset (Year 6 only), and Sandwell (Reception only). Baseline data from a previously described cohort study were used in this analysis, the protocol for which has been previously published. ${ }^{11}$ Questionnaires were distributed to parents on the day of the child's NCMP measurement but before they received weight feedback. Parents were asked to state whether they believed their child was underweight, healthy weight, overweight, or very overweight. PCT records provided the child's age, sex, ethnic group, school year, NCMP nursemeasured height and weight, and local area deprivation quintile from the Index of Multiple Deprivation (IMD) 2007.12

\section{Primary outcome: parental cut-offs}

A multinomial model of parental perceived weight status was used, with three categories: underweight, healthy weight, and overweight las the number of parents reporting their child as very overweight was low $[n=4]$, the very overweight and overweight categories were combined). A proportional odds model was rejected in favour of a multinomial model, as the proportional odds assumption was violated. Coefficients did not vary importantly between the two models. The only independent variable was the child's BMI /calculated from measured weight and height), expressed as a z-score from the UK90 reference population to improve model fit. ${ }^{9}$ The z-scores were calculated directly from centiles, and represent the distance of a child's BMI from the reference population mean. Standard errors were adjusted for school-level clustering. Models were initially created by school year (reception or Year 6) within sexes. A lack of variability in the estimates across models suggested that an unadjusted model was appropriate. A post hoc sensitivity analysis showed BMI centile cut points for underweight and overweight in an analogous model adjusted for school year and sex were 0.8 195\% confidence interval $[\mathrm{Cl}]=0.5$ to 1.7) and 99.9 (95\% $\mathrm{Cl}=99.4$ to 99.9), respectively.

Using an unadjusted multinomial logistic regression model, cut scores were obtained that represent the points at which a child's z-score is equally likely to be classified into two adjoining weight status categories, that BMI z-scores were identified at which a child is equally as likely to be described as underweight as healthy weight, or healthy weight as overweight, by their parents. Published equations were used to derive ordinal cut-offs from the univariate multinomial model, ${ }^{13}$ and verified against the plotted predicted conditional probabilities. To ease interpretation, $z$-scores were presented as centiles in the results.

\section{Secondary outcome: what predicts misclassification?}

Two types of misclassification were defined:

- parent-reported weight status lunderweight, healthy, overweight, or very overweight) as an underestimate of true weight status (defined using population-level BMI cut-offs), and;

- parent-reported weight status as an overestimation.

The effects of ethnic group, IMD quintile, sex, and school year on underestimating weight status versus correctly or overestimating weight status were explored using logistic regression. Overestimating weight status versus correctly or underestimating weight status was explored in a separate, otherwise analogous, model. Standard errors were adjusted for school-level clustering. Because of the low number of overestimations potentially introducing bias, ${ }^{14}$ the reported results for overestimation were derived from an analogous exact logistic regression model. 


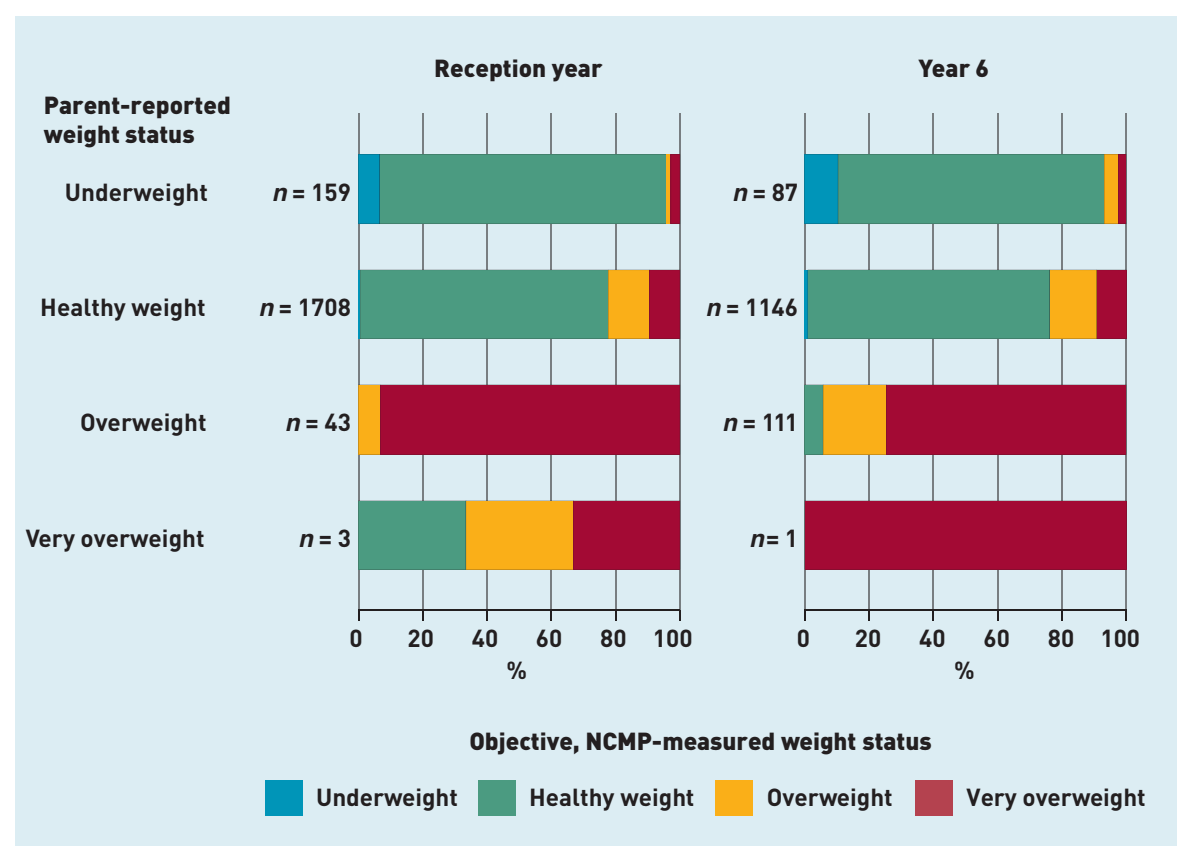

Figure 1. Categorisation of children's weight status by National Child Measurement Programme (NCMP)-measurement against parent-reported category.

Figure 2. Distribution of body mass index centiles in 2976 children, with bars colour coded by parent-reported weight status within each decile.
Central estimates for overestimation were similar to the standard maximum likelihood estimate (MLE) logistic regression.

For the secondary analysis, standard population level lunderweight <2nd centile, overweight $>85$ th centile, and very overweight >95th centile), rather than clinical, weight status cut-offs were compared with parentreported categories. Parents are more likely to have been exposed to these population cut-offs before receiving NCMP feedback on their child's weight status. Stata lversion 12.1) was used for all analyses.

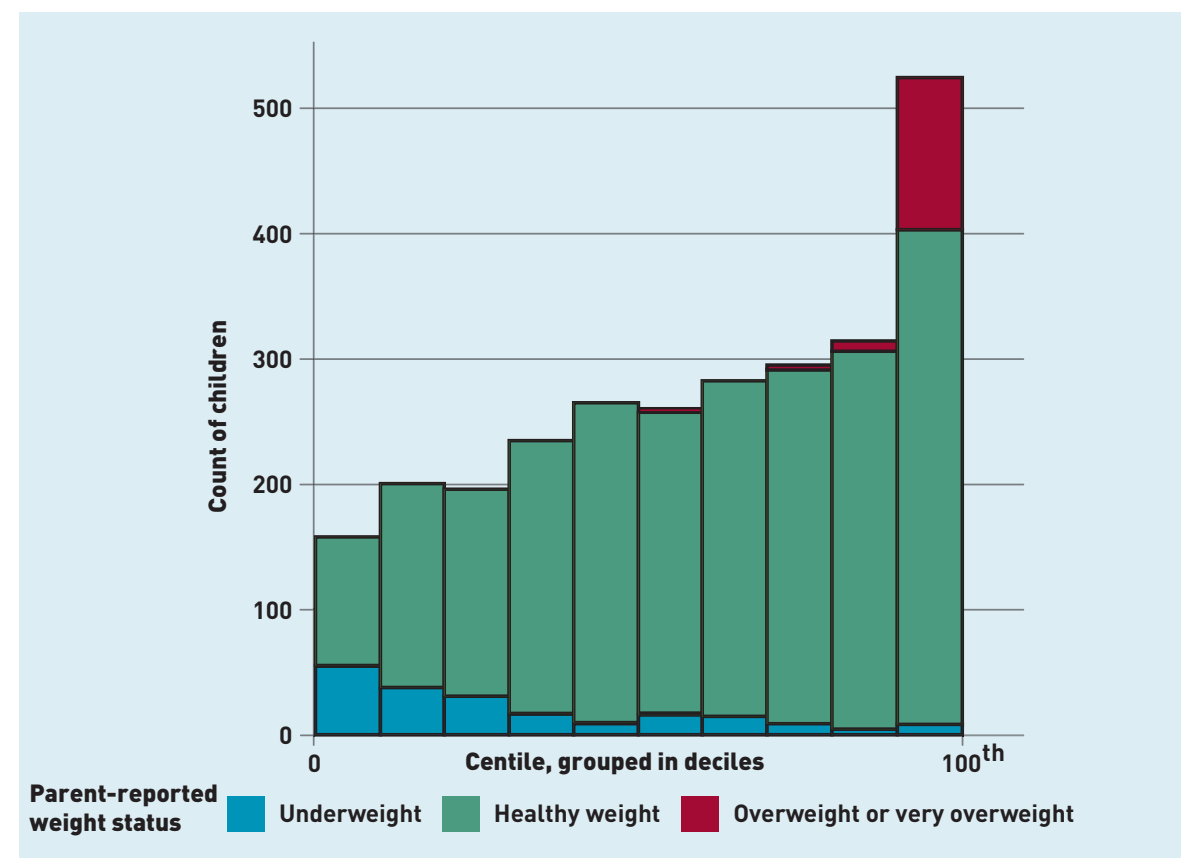

\section{RESULTS}

In total, 3397 parents responded to the questionnaire (response rate 15\% of all children in the five PCTs). Of these, 139 parents did not complete the question asking about their child's weight status, and 282 children were not present on PCT records. This left 2976 (88\%) children with both parent-reported weight status and a valid NCMP measurement. Responders to the questionnaire were predominantly mothers (87\%), followed by fathers (12\%), and step- or grandparents (1\%). Overall, $68 \%$ of responders classified their child into the correct weight status category (Figure 1).

The distribution of BMI centiles was skewed, with $20 \%$ of children in the highest decile (Figure 2). Four parents described their child as being very overweight, despite 369 being very overweight according to the BMI cut-offs (Figure 1). Using the three level classifications lunderweight, healthy weight, and overweight), $72 \%$ of parents correctly classified their child.

\section{What do parent-reported cut-offs look like?}

The point at which a parent was equally likely to recognise underweight as healthy weight was when their child had a BMI at the 0.8 th $(95 \% \mathrm{Cl}=0.4$ to 1.1$)$ centile. $\mathrm{A}$ parent became more likely to classify their child as overweight, rather than healthy weight, when the child had a $\mathrm{BMI} \geq 99.7 \mathrm{th}$ centile $(95 \% \mathrm{Cl}=99.3$ to 99.9$)$ (Figure 3). As an example, for a child with a BMI at the 98th centile (very overweight), it is estimated that the chance the parent would classify their child as healthy weight is $80 \%$ $(95 \% \mathrm{Cl}=76$ to 83$)$ and there is a $20 \% 195 \%$ $\mathrm{Cl}=16$ to 23) chance of overweight or very overweight. While at the 99.7th centile, a parent has an equal chance of classifying their child as healthy or overweight.

\section{What predicts misclassification?}

In total, 915 (31\%) parents underestimated and $25(<1 \%)$ overestimated their child's weight status. Parents were more likely to underestimate their child's weight status if they were black $\operatorname{OR} 1.5,95 \% \mathrm{Cl}=1.1$ to 2.1), South Asian (OR 1.6, $95 \% \mathrm{Cl}=1.3$ to 2.0), male (OR $1.3,95 \% \mathrm{Cl}=1.1$ to 1.6$)$, or older (OR 1.3, 95\% Cl=1.1 to 1.5) (Table 1). Parents from less deprived areas were less likely to underestimate their child's weight status (IMD quintile, OR 0.8, 95\% $\mathrm{Cl}=0.75$ to 0.9$)$. Overestimation of weight status was more likely for 10-11-year-olds than 4-5-year-olds (OR 3.1, 95\% Cl=1.2 to 8.5). Ethnic group, deprivation, and sex 


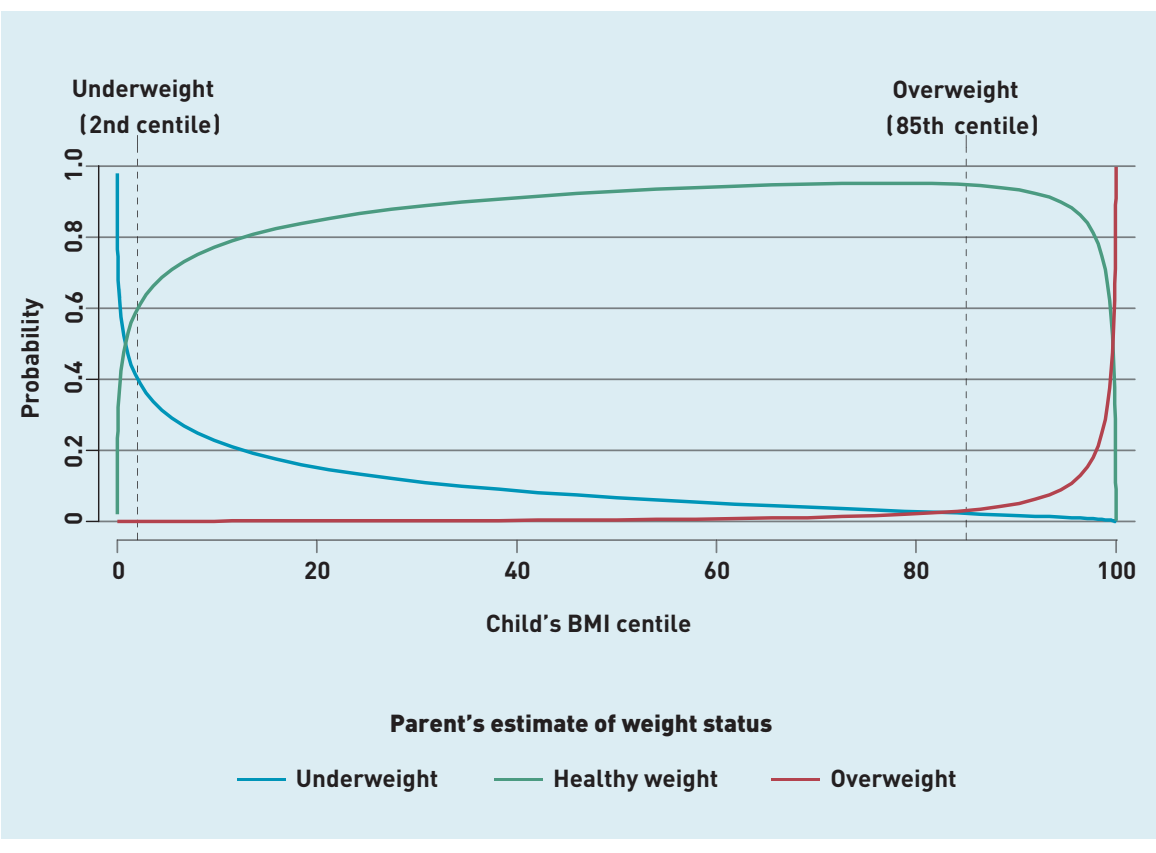

Figure 3. Probability of child's caregiver classifying their weight as underweight, healthy, or overweight against National Child Measurement Programme (NCMP)-measured body mass index centile in 2976 British children. did not predict parental overestimation of weight status.

\section{DISCUSSION}

\section{Summary}

Child BMI centile cut-offs were modelled based on parent-reported weight status, and it was found that parents become more likely to classify their children as underweight when they are at the 0.8th centile or below, and overweight at the 99.7th centile or above. These values differ greatly from the BMI centile cut-offs for underweight (2nd centile) and overweight (85th) used widely in the literature,

\section{Table 1. Predictors of a parent incorrectly classifying their child's weight status}

Parent underestimates weight status

\section{White}

Black

Asian

Mixed/other

IMD quintile (one quintile less deprived)

Male

Year 6, age 10-11 years (versus Reception, age 4-5 years

\section{Parent overestimates weight status ${ }^{a}$}

\begin{tabular}{lc}
\hline White & Reference \\
Black & 4.1 (0.8 to 17.0$)$ \\
Asian & 2.9 (0.9 to 9.5) \\
Mixed/other & 3.0 (0.7 to 11.0) \\
\hline IMD quintile (one quintile less deprived) & 0.9 (0.6 to 1.2) \\
\hline Male & 0.9 (0.4 to 2.2) \\
\hline Year 6, age 10-11 years (versus Reception, age 4-5 years) & $3.1(1.2$ to 8.5$)$ \\
\hline
\end{tabular}

Year 6, age 10-11 years (versus Reception, age $4-5$ years)

${ }^{a}$ Results from exact logistic regression. $I M D=$ Index of Multiple Deprivation. $O R=$ odds ratio government guidelines, and public health messages. Parents were more likely to underestimate a child's weight if the child was black or South Asian (versus white), male, more deprived, or the child was older lage 10-11 years versus 4-5 years). Few parents overestimated their child's weight status, but overestimation was more common among parents of older children.

\section{Strengths and limitations}

A large, ethnically and demographically diverse population from five PCTs is represented in this study. Heights and weights for calculation of $\mathrm{BMI}$ were measured by trained school nurses as part of the NCMP, which has an exceptionally high participation rate $193 \%$ of target population in 2011-2012). ${ }^{8}$ Although there is concern over using BMl weight status cut-offs for adiposity at an individual level, ${ }^{15}$ pragmatically, the $>98$ th centile BMI cutoff has moderate sensitivity (71\%) and high specificity (98\%) relative to clinically measured impedance, ${ }^{15}$ limiting the number of children being falsely identified as very overweight. Whereas previous studies have found a divergence between parental perception of weight status and objectively measured weight status, $, 0,10,16-18$ this study is the first to quantify the disparity between objective cut-offs and cut-offs derived from parental perceptions.

This study assessed parental perception of weight status, and misclassification was explored through comparisons with objectively assessed weight status. As such, it cannot be concluded if the cutoffs reported are specific to parents, or also present in subjective assessments by health professionals.

This study was limited by the low number of parents with very overweight children classifying their children as very overweight, meaning both overweight and very overweight were collapsed into a single overweight category. Few parents overestimated their child's weight status, and, to reduce bias, methods were used that inflated variance of the estimates, potentially masking smaller associations. Data on parental weight perceptions were collected by postal questionnaire.

The low response rate for the questionnaire introduces the potential for non-response bias: responders to the questionnaire were more likely to be from white ethnic groups and less deprived than the target population (Table 2). The sample characteristics suggest that they were slightly more likely to correctly identify their child's weight status. Although no 


\section{Table 2. Summary of NCMP population and analysis sample}

\begin{tabular}{lcc} 
& Analysis sample & $\begin{array}{c}\text { Non-responders from } \\
\text { the NCMP population in } \\
\text { the five included PCTs }\end{array}$ \\
\hline Reception, $n$ & 1422 & 8068 \\
\hline Mean age, years (SD) & $5.1(0.31)$ & $5.1(0.34)$ \\
\hline Female, \% & 49.0 & 47.9 \\
\hline White, \% & 57.8 & 49.0 \\
\hline Underweight: $<$ 2nd centile, \% & 0.8 & 1.1 \\
Overweight: $>$ 85th centile, \% & 12.4 & 12.3 \\
Very overweight: $>95$ th centile, \% & 9.9 & 10.8 \\
\hline In most deprived IMD quintile, \% & 18.8 & 22.6 \\
\hline Year 6, $n$ & 1312 & 8394 \\
\hline Mean age,years (SD) & $11.1(0.32)$ & $11.1(0.34)$ \\
\hline Female, \% & 52.3 & 48.5 \\
\hline White, \% & 67.4 & 59.7 \\
\hline Underweight: $<2$ nd centile, \% & 1.5 & 1.7 \\
Overweight: $>$ 85th centile, \% & 14.4 & 14.4 \\
Very overweight: $>$ 95th centile, \% & 13.9 & 20.8 \\
\hline In most deprived IMD quintile, \% & 14.9 & 18.3
\end{tabular}

$I M D=$ Index of Multiple Deprivation. NCMP = National Child Measurement Programme. PCT = primary care trust.

\section{Funding}

This article presents independent research funded by the National Institute for Health Research (NIHR) in England under its Programme Grants for Applied Research programme (RP-PG-0608-10035). The views expressed in this publication are those of the authors and do not necessarily reflect those of the NHS, the NIHR, or the Department of Health. Sonia Saxena is funded by an NIHR postdoctoral fellowship. The funder of the study had no role in study design, data collection, data analysis, interpretation of data, or writing of the manuscript.

\section{Ethical approval}

Obtained from the London School of Hygiene and Tropical Medicine Ethics Committee.

\section{Provenance}

Freely submitted; externally peer reviewed.

\section{Competing interests}

The authors have declared no competing interests.

\section{Acknowledgements}

We thank the primary care trusts, schools, parents, and children who participated in this study.

\section{Discuss this article}

Contribute and read comments about this article: bjgp.org/letters substantial changes were seen in the modelled cut-offs stratifying for age and sex, these analyses were underpowered because of small numbers of parents identifying their children as underweight and overweight.

\section{Comparison with existing literature}

The authors are not aware of other studies that have derived BMI cut-offs based on parent-perceived weight status; however, previous studies have shown that parents are more likely to misclassify their child towards the healthy weight category. ${ }^{6,10}$ In contrast to a literature review of 52 studies that found parents were more likely to misclassify children aged 2-6 years than older children, ${ }^{6}$ this study found that older children (10-11-year-olds) were more likely to be misclassified than younger children (5-6 years). As has been noted in the literature, ${ }^{16}$ this study found that parents were more likely to underestimate a boy's weight status, potentially highlighting sex-specific norms for body composition and appearance. Currently, little is known about parental perceptions of child obesity in Asian communities. In a recent survey of parents with children aged 4-16 years in Liverpool, parental beliefs and attitudes about childhood obesity differed significantly by ethnicity, but this study did not compare parental perceptions with children's measured weight status. ${ }^{19}$ In a more methodologically robust study, selfreported weight misclassification did not vary by ethnic group among 11-14-year- olds in a community health survey of an ethnically-diverse population in London. ${ }^{20}$

Although factors associated with misclassification of weight status have been identified, currently there is limited evidence suggesting mechanisms to explain these differences. Potential explanations may be fear of being judged, unwillingness to label a child as overweight, and shifting perceptions of normal weight because of increases in body weight at a societal level. ${ }^{16}$

\section{Implications for practice}

Parental involvement aids in the promotion of healthy weight maintenance in children. ${ }^{21}$ A Cochrane Review of interventions for preventing child obesity identified parental support to enact healthy lifestyle changes at home as a promising strategy for weight reduction. ${ }^{21}$ Evidence suggests that parents who recognise their child's weight status are more likely to perceive potential health risks. ${ }^{22}$ Although BMl cut-offs in childhood can be considered arbitrary in some ways lbecause they are based on population norms rather than specified health risks associated with them), childhood overweight and obesity have been shown to predict adult weight status and the health problems associated with excess weight in adulthood. ${ }^{1,3,5}$ The parents of some children who are most at risk of health consequences of being overweight, including South Asian boys, appear from these study findings to be least able to detect weight problems in their child. ${ }^{23}$ Parental perceived weight status cut-offs are judgements based on a diverse range of values, perceptions, and beliefs. If parents are unable to accurately classify their own child's weight, they may not be willing or motivated to enact changes to the child's environment that promote healthy weight maintenance.

This study has demonstrated the extreme divergence between clinical and parental classifications of obesity. This discrepancy in perceived weight status is important for policy makers and clinicians to consider in their approaches to obesity prevention; weight management interventions targeted at the parents of overweight children are unlikely to register with the intended audience if few parents consider their child to be overweight. Measures that decrease the gap between parental perceptions of child weight status and BMI cut-offs used by medical professionals may help parents better understand the health risks associated with being overweight and increase uptake of healthier lifestyles. ${ }^{17,22}$ 


\section{REFERENCES}

1. Pinhas-Hamiel O, Zeitler P. 'Who is the wise man? - The one who foresees consequences'. Childhood obesity, new associated comorbidity and prevention Prev Med 2000; 31(6) 702-705.

2. de Onis M, Blössner M, Borghi E. Global prevalence and trends of overweight and obesity among preschool children. Am J Clin Nutr 2010; 92(5): 1257-1264.

3. Jackson-Leach R, Lobstein T. Estimated burden of paediatric obesity and co-morbidities in Europe. Part 1. The increase in the prevalence of child obesity in Europe is itself increasing. Int J Pediatr Obes 2006; 1(1): 26-32.

4. Health and Social Care Information Centre and Public Health England. National Child Measurement Programme: England, 2012/13 school year. Leeds: HSIC 2013

5. Reilly JJ, Kelly J. Long-term impact of overweight and obesity in childhood and adolescence on morbidity and premature mortality in adulthood: systematic review. Int J Obes 2011; 35(7): 891-898.

6. Rietmeijer-Mentink M, Paulis WD, van Middelkoop M, et al. Difference between parental perception and actual weight status of children: a systematic review. Matern Child Nutr 2013; 9(1): 3-22.

7. Parry LL, Netuveli G, Parry J, Saxena S. A systematic review of parental perception of overweight status in children. J Ambul Care Manage 2008; 31(3): 253-268.

8. Public Health England. National Child Measurement Programme: operational guidance for the 2013/14 school year. London: Public Health England, 2013.

9. Cole TJ, Freeman JV, Preece MA. Body mass index reference curves for the UK 1990. Arch Dis Child 1995; 73(1): 25-29.

10. Maximova K, McGrath JJ, Barnett T, et al. Do you see what I see? Weight status misperception and exposure to obesity among children and adolescents. Int J Obes 2008; 32(6): 1008-1015

11. Falconer $\mathrm{CL}$, Park M, Skow A, et al. Scoping the impact of the national child measurement programme feedback on the child obesity pathway: study protocol. BMC Public Health 2012; 12: 783.

12. Department for Communities and Local Government. The English Indices of Deprivation 2007: summary. London: Department for Communities and Local Government, 2007.

13. Bersabé R, Rivas T. A general equation to obtain multiple cut-off scores on a test from multinomial logistic regression. Span J Psychol 2010; 13(1): 494-502.

14. Mehta CR, Patel NR. Exact logistic regression: Theory and examples. Stat Med 1995; 14(19): 2143-2160

15. Reilly JJ, Dorosty AR, Emmett PM. Identification of the obese child: adequacy of the body mass index for clinical practice and epidemiology. Int J Obes Relat Metab Disord 2000; 24(12): 1623-1627.

16. Doolen J, Alpert PT, Miller SK. Parental disconnect between perceived and actual weight status of children: a metasynthesis of the current research. J Am Acad Nurse Pract 2009; 21(3): 160-166.

17. Garrett-Wright D. Parental perception of preschool child body weight. J Pediatr Nurs 2011; 26(5): 435-445

18. Wake M, Canterford $L$, Hardy P, Ukoumunne OC. At what BMl are parents of pre-schoolers concerned? National cross-sectional study. Int J Pediatr Obes 2011; 6(5-6): 499-501.

19. Trigwell J, Watson P, Murphy R, et al. Ethnic differences in parental attitudes and beliefs about being overweight in childhood. Health Educ J 2014; 73(2): 179-191.

20. Viner RM, White B, Barrett $\mathrm{T}$, et al. Assessment of childhood obesity in secondary care: OSCA consensus statement. Arch Dis Child 2012; 97(3): 98-105.

21. Summerbell $C$, Waters $E$, Edmunds $L$, et al. Interventions for preventing obesity in children. Cochrane Database Syst Rev2011; 12: CD001871.

22. Park MH, Falconer $\mathrm{CL}$, Saxena S, et al. Perceptions of health risk among parents of overweight children: a cross-sectional study within a cohort. Prev Med 2013; 57(1): 55-59.

23. Saxena S, Ambler G, Cole TJ, Majeed A. Ethnic group differences in overweigh and obese children and young people in England: cross sectional survey. Arch Dis Child 2004: 89(1): 30-36. 